\title{
Correction: Surveillance of pyrazinamide susceptibility among multidrug-resistant Mycobacterium tuberculosis isolates from Siriraj Hospital, Thailand
}

\author{
Jirarut Jonmalung ${ }^{1}$, Therdsak Prammananan ${ }^{2,4}$, Manoon Leechawengwongs ${ }^{3,4}$, Angkana Chaiprasert $^{1,4^{*}}$
}

\section{Correction}

After galley proof of the manuscript, we found three mistakes of the nucleotide positions (G222C, G364A and C520T) and codon numbers (Gly74Arg, Gly122Ser and Thr174Ile) that have to be corrected but it was unable to make any change because the publication of this work is on going [1]. After revision, Table two (Table 1 in this manuscript) and some information in the discussion part were changed. There were only 5 novel mutation types found in this study, consisting of 2 nucleotide substitutions (Leu27Pro and Thr174Ile), 2 nucleotide insertions ( $G$ insertion between nucleotide 411 and 412 and GG insertion between nucleotide 520 and 521), and 1 nonsense mutation at Glu127.

We regret any inconvenience that the mistake might have caused. We wish to thank Dr. Claudio Köser, Department of Genetics, University of Cambridge, for bringing this matter to our attention.

\section{Author details \\ 'Department of Microbiology, Faculty of Medicine Siriraj Hospital, Mahidol University, Bangkok, 10700, Thailand. ${ }^{2}$ National Center for Genetic Engineering and Biotechnology, National Science and Technology, Development Agency, Ministry of Science and Technology, Pathumthani 12120, Thailand. ${ }^{3}$ Vichaiyut Hospital, Setsiri Road, Bangkok 10400, Thailand. ${ }^{4}$ Drug-Resistant Tuberculosis Research Fund, Siriraj Foundation, Bangkok 10700, Thailand.}

Received: 28 October 2010 Accepted: 9 November 2010 Published: 9 November 2010

\section{Reference}

1. Jonmalung J, Prammananan T, Leechawengwongs M, Chaiprasert A: Surveillance of pyrazinamide susceptibility among multidrug-resistant

\footnotetext{
* Correspondence: siacp@mahidol.ac.th

'Department of Microbiology, Faculty of Medicine Siriraj Hospital, Mahidol University, Bangkok, 10700, Thailand
}

Full list of author information is available at the end of the article
Mycobacterium tuberculosis isolates from Siriraj Hospital, Thailand. BMC Microbiology 2010, 10:223.

\section{doi:10.1186/1471-2180-10-278}

Cite this article as: Jonmalung et al:: Correction: Surveillance of pyrazinamide susceptibility among multidrug-resistant Mycobacterium tuberculosis isolates from Siriraj Hospital, Thailand. BMC Microbiology 2010 10:278.
Submit your next manuscript to BioMed Central and take full advantage of:

- Convenient online submission

- Thorough peer review

- No space constraints or color figure charges

- Immediate publication on acceptance

- Inclusion in PubMed, CAS, Scopus and Google Scholar

- Research which is freely available for redistribution

Submit your manuscript at www.biomedcentral.com/submit
C Biomed Central 
Table 1 Results of pncA gene sequencing of $150 \mathrm{M}$. tuberculosis clinical isolates

\begin{tabular}{|c|c|c|c|c|}
\hline \multirow[b]{2}{*}{ M. tuberculosis strains (no. of isolates) } & \multirow[b]{2}{*}{ MGIT 960} & \multirow[b]{2}{*}{ PZase assay } & \multicolumn{2}{|l|}{ pncA mutation } \\
\hline & & & Nucleotide change & Amino acid change \\
\hline Susceptible (46) & S & + & wild-type & no \\
\hline Susceptible (1) & S & + & T92G & \|le31Ser \\
\hline Susceptible (2) & R & + & wild-type & wild-type \\
\hline Susceptible (1) & R & + & T92C & \|le31Thr \\
\hline MDR-TB (42) & S & + & wild-type & wild-type \\
\hline MDR-TB (9) & S & + & T92C & lle31Thr \\
\hline \multirow[t]{23}{*}{ MDR-TB (34) } & R & - & $A(-11) G(1)$ & no \\
\hline & & & $A(-11) C(1)$ & no \\
\hline & & & T56G (1) & Leu19Arg \\
\hline & & & T80C (1) & Leu27Pro \\
\hline & & & T92G (2) & Ile31Ser \\
\hline & & & $\mathrm{T} 104 \mathrm{C}(1)$ & Leu35Pro \\
\hline & & & $\mathrm{T} 134 \mathrm{C}(1)$ & Val45Ala \\
\hline & & & G136T (1) & Ala46Ser \\
\hline & & & T199C (1) & Ser67Pro \\
\hline & & & C211G (8) & His71Asp \\
\hline & & & G215A (1) & Cys72Tyr \\
\hline & & & G289A (3) & Gly97Ser \\
\hline & & & C312G (2) & Ser104Arg \\
\hline & & & G322C (1) & Gly108Arg \\
\hline & & & G373T (1) & Val125Phe \\
\hline & & & G379T (1) & Glu 127 Stop \\
\hline & & & G394A (1) & Gly132Ser \\
\hline & & & G insertion b/w 411-412 (1) & \\
\hline & & & T416G (1) & Val 139 Gly \\
\hline & & & C425T (1) & Thr 142 Met \\
\hline & & & G436A (1) & Ala $146 \mathrm{Thr}$ \\
\hline & & & GG insertion b/w 520-521 (1) & \\
\hline & & & C530T (1) & Thr 177 Ile \\
\hline MDR-TB (11) & R & + & wild-type & no \\
\hline \multirow[t]{2}{*}{ MDR-TB (4) } & $\mathrm{R}$ & + & T92C (3) & Ile31Thr \\
\hline & & & T92G (1) & Ile31Ser \\
\hline
\end{tabular}

